\title{
Fam107A is differentially expressed in non-small cell lung cancer and associates with
} patient survival.

Shahan Mamoor ${ }^{1}$

1shahanmamoor@gmail.com

East Islip, NY USA

Non-small cell lung cancer (NSCLC) is the leading cause of cancer death in the United States ${ }^{1}$. We mined published microarray data $2,3,4$ to identify differentially expressed genes in NSCLC. We found that the gene encoding family with sequence similarity 107A, Fam107A was among those whose expression was most different in human NSCLC tumors as compared to the lung. Fam107A expression levels were significantly decreased in NSCLC tumors as compared to the lung, and lower expression of Fam107A in patient tumors was significantly associated with worse overall survival. Fam107A may be important for initiation or progression of non-small cell lung cancer in humans.

Keywords: Fam107A, NSCLC, non-small cell lung cancer, systems biology of NSCLC, targeted therapeutics in NSCLC. 
In 2016, lung cancer resulted in the death of 158,000 Americans; $81 \%$ of all patients diagnosed with lung cancer will expire within 5 years 5 . Non-small cell lung cancer (NSCLC) is the most common type of lung cancer, diagnosed in $84 \%$ of patients with lung cancer, and $76 \%$ of all patients with NSCLC will expire within 5 years ${ }^{5}$. The rational development of targeted therapeutics to treat patients with NSCLC can be supported by an enhanced understanding of fundamental transcriptional features of NSCLC tumors. To discover genes associated with NSCLC tumors in an unbiased fashion and at the systems-level, we mined independently published microarray data $2,3,4$ to compare global gene expression profiles of NSCLC tumors to that of the normal lung. We found recurrent and significant differential expression of the gene encoding Fam107A in adenocarcinoma tumors from patients with NSCLC, suggesting Fam107A may be important for NSCLC tumor initiation or progression.

\section{Methods}

We utilized microarray datasets GSE74706², GSE4345832, GSE3353244 and for this differential gene expression analysis of NSCLC tumors in conjunction with GEO2R. GSE74706 was generated using Agilent-026652 Whole Human Genome Microarray 4x44K v2 technology; for this analysis, we used $n=18$ control lung tissue and $n=10$ NSCLC tumors, and the analysis was performed using platform GPL13497. GSE43458 was generated using Affymetrix Human Gene 1.0 ST Array technology; for this analysis, we used with $n=30$ control lung tissue and $n=80$ NSCLC tumors, and the analysis was performed using platform GPL6244. GSE33532 was generated using Affymetrix Human Genome U133 Plus 2.0 Array technology; for this analysis, 
we used $n=20$ control lung tissue and $n=10$ NSCLC tumors, and the analysis was performed using platform GPL570. All tumors utilized for differential gene expression analysis here were of the adenocarcinoma type.

The Benjamini and Hochberg method of $p$-value adjustment was used for ranking of differential expression but raw $p$-values were used to assess statistical significance of global differential expression. Log-transformation of data was auto-detected, and the NCBI generated category of platform annotation was used. A statistical test was performed to evaluate whether Fam107A expression was significantly between normal lung tissue and NSCLC tumors using a two-tailed, unpaired t-test with Welch's correction. We used PRISM for all statistical analyses of differential gene expression in NSCLC tumors (Version 8.4.0)(455). For KaplanMeier survival analysis, we used the Kaplan-Meier plotter online tool ${ }^{6}$ for correlation of Fam107A mRNA expression levels with overall survival in $n=1925$ non-small cell lung cancer patients.

\section{Results}

We harnessed the power of multiple, independently published microarray datasets $2,3,4$ to discover in an unbiased fashion and at the transcriptome-level the most striking gene expression features of NSCLC tumors.

Fam107A is differentially expressed in non-small cell lung cancers.

We found significant differential expression of the gene encoding family with sequence

3 OF 17 
similarity 107A, Fam107A, in NSCLC tumors when compared to the lung'2 (Table 1). When sorting each of the transcripts measured based on significance of difference in expression of Fam107A between NSCLC tumors and the normal lung, in this dataset, Fam107A ranked 27 out of 34183 total transcripts (Table 1). Differential expression of Fam107A in NSCLC tumors was statistically significant (Table $1 ; p=5.6 \mathrm{E}-14)$.

We queried a second microarray dataset ${ }^{3}$ to determine if we could validate differential expression of Fam107A in non-small cell lung cancers (Table 2). We found significant differential expression of Fam107A in NSCLC tumors of the adenocarcinoma type when compared to the normal lung (Table 2). When sorting each of the transcripts measured based on significance of difference in expression of Fam107A between NSCLC tumors and the normal lung, Fam107A ranked 72 of 33252 total transcripts (Table 2). Differential expression of Fam107A in NSCLC tumors was statistically significant (Table $2 ; p=3.54 \mathrm{E}-25$ ).

Analysis of a third microarray dataset ${ }^{4}$ again revealed significant differential expression of Fam107A in NSCLC tumors of the adenocarcinoma type. When sorting each of the transcripts measured based on significance of difference in expression of Fam107A between NSCLC tumors and the normal lung, Fam107A ranked 26 out of 25906 total transcripts (Table 3). Differential expression of Fam107A in NSCLC tumors was statistically significant (Table 3; $p=4.44 \mathrm{E}-17)$.

Fam107A is expressed at significantly lower levels in NSCLC tumors as compared to the lung. We obtained exact mRNA levels for Fam107A from NSCLC tumors and from the lung to 
directly compare Fam107A expression between tumor and control lung tissue and assess for statistical significance. Fam107A was expressed at significantly lower levels in NSCLC tumors as compared to the normal lung in each dataset queried, respectively (Figure 1: $p<0.0001$, Figure 2: $p<0.0001$, Figure 3: $p<0.0001$ ). We calculated a mean fold change of $0.8413 \pm 0.0528$ and $0.5990 \pm 0.0867$ in Fam107A expression when comparing NSCLC tumors to the lung (Table 2 and Table 3 , respectively).

Fam107A expression in NSCLC tumors correlates with overall survival.

We performed Kaplan-Meier survival analysis using Fam107A mRNA expression in NSCLC tumors coupled with paired overall survival data from each patient, in 1925 NSCLC patients in total, to determine whether Fam107A tumor expression was correlated with survival outcomes in NSCLC. We found that patients whose tumors expressed lower levels of Fam107A possessed significantly shorter overall survival than patients with high tumor expression of Fam107A (Figure 4). Median overall survival (OS) of patients in the low expression cohort was 56.5 months, while median OS in patients in the high Fam107A expression cohort was 103 months (Table 4); this difference in median OS based on Fam107A tumor expression in NSCLC was statistically significant (Figure 4; logrank $p$-value: $2.9 \mathrm{e}-10$; hazard ratio: $0.63(0.55-0.73)$; false discovery rate $=0.01$ ).

Thus, blind comparative transcriptome analysis of non-small cell lung cancers revealed differential expression of transcripts encoded by the Fam107A gene as among the most significant transcriptional features of NSCLC tumors, and Fam107A expression was significantly 
correlated with patient outcomes, as patients with lower tumor expression of Fam107A possessed significantly worse overall survival.

\section{Discussion}

Fam107A, also known as TU3A, was identified in an investigation of an area of genomic loss in renal cell carcinoma on chromosome $3^{7}$. A gene product encoding 144 amino acids was isolated, translated from a transcript of 3 kilobases with ubiquitous expression. Fam107A contains a domain of unknown function at the amino-terminus termed DUF1151, a centrally located nuclear localization signal, and two motifs within the DUF1151: a histidine-arginineglutamic acid (HRE) sequence, and a proline-glutamic acid (PE) motif ${ }^{8}$. A study of the brain, specifically of the radial glia found that FAM107A was expressed in the ventricular zone at gestational weeks 13.5, and that its expression was up-regulated in outer radial glia cells9. Fam107A is described as being perturbed at the genomic level or at the level of expression in human cancers. Of five renal cell carcinoma cell lines examined in the study in which Fam107A was identified, two had lost expression of Fam107A/TU3A7 . Whole exome sequencing of a family with five members who were diagnosed with nodular sclerosis classical Hodgkin's lymphoma revealed a missense mutation in Fam107A resulting in a mutation of arginine at position 76 to glutamine, and patient-derived lymphoblastoid cell lines revealed lack of Fam107A expression ${ }^{10}$. Fam107A (also known as DRR1) was also identified as a differentially expressed gene in a study of low-grade gliomas that recurred as secondary glioblastomas ${ }^{11}$. Fam107A expression was significantly lower in secondary glioblastomas and in 
primary glioblastomas when compared to diffuse astrocytomas ${ }^{11}$. Hyper-methylation of the Fam107A locus was found in 22 out of 53 renal cell carcinoma cancers and Fam107A hypermethylation correlated with worse disease-specific survival ${ }^{12}$. We found one study describing a role for Fam107A in non-small cell lung cancer, with decreased expression of Fam107A in NSCLC tumors as compared to normal adjacent lung tissue, and with promoter methylation of Fam107A detectable in a minority of cases surveyed ${ }^{13}$. Thus, Fam107A contains a domain of unknown function, DUF1151, its expression is lost or decreased in a number of cancers and hyper-methylation of the Fam107A locus has also been documented in cancer.

We found, by mining multiple independently published microarray datasets, that Fam107A was among the genes most differentially expressed in the primary tumors of patients with non-small cell lung cancer; Fam107A was expressed at significantly lower levels in NSCLC tumors as compared to the lung, and decreased expression of Fam107A was significantly associated with worse overall survival in NSCLC patients. Fam107A may be of relevance to the biology of tumor initiation or progression in patients with NSCLC of the adenocarcinoma type, the most common type of the leading cause of cancer death in the United States and worldwide. 


\section{References}

1. Siegel, R.L., Miller, K.D. and Jemal, A., 2019. Cancer statistics, 2019. CA: a cancer journal for clinicians, 69(1), pp.7-34.

2. Kabbout, M., Garcia, M.M., Fujimoto, J., Liu, D.D., Woods, D., Chow, C.W., Mendoza, G., Momin, A.A., James, B.P., Solis, L. and Behrens, C., 2013. Ets2 mediated tumor suppressive function and met oncogene inhibition in human non-small cell lung cancer. Clinical cancer research, 19(13), pp.3383-3395.

3. Meister, M., Belousov, A., Xu, E.C., Schnabel, P., Warth, A. and Hoofmann, H., 2014. Intratumor heterogeneity of gene expression profiles in early stage non-small cell lung cancer. J Bioinf Res Stud, 1, p.1.

4. Marwitz, S., Depner, S., Dvornikov, D., Merkle, R., Szczygieł, M., Müller-Decker, K., Lucarelli, P., Wäsch, M., Mairbäurl, H., Rabe, K.F. and Kugler, C., 2016. Downregulation of the TGF $\beta$ pseudoreceptor BAMBI in non-small cell lung cancer enhances TGF $\beta$ signaling and invasion. Cancer research, 76(13), pp.3785-3801.

5. Lung Cancer - Non-Small Cell: Statistics. https://www.cancer.net/cancer-types/lung-cancernon-small-cell/statistics.

6. Gyorffy, B., Surowiak, P., Budczies, J. and Lanczky, A., 2013. Online survival analysis software to assess the prognostic value of biomarkers using transcriptomic data in non-smallcell lung cancer. PloS one, 8(12), pp.e82241-e82241.

7. Nakajima, H. and Koizumi, K., 2014. Family with sequence similarity 107: A family of stress responsive small proteins with diverse functions in cancer and the nervous system. Biomedical reports, 2(3), pp.321-325.

8. Yamato, T., Orikasa, K., Fukushige, S., Orikasa, S. and Horii, A., 1999. Isolation and characterization of the novel gene, TU3A, in a commonly deleted region on 3p14. 3 $\rightarrow$ p14. 2 in renal cell carcinoma. Cytogenetic and Genome Research, 87(3-4), pp.291-295.

9. Pollen, A.A., Nowakowski, T.J., Chen, J., Retallack, H., Sandoval-Espinosa, C., Nicholas, C.R., Shuga, J., Liu, S.J., Oldham, M.C., Diaz, A. and Lim, D.A., 2015. Molecular identity of human outer radial glia during cortical development. Cell, 163(1), pp.55-67.

10.Lawrie, A., Han, S., Sud, A., Hosking, F., Cezard, T., Turner, D., Clark, C., Murray, G.I., Culligan, D.J., Houlston, R.S. and Vickers, M.A., 2018. Combined linkage and association analysis of classical Hodgkin lymphoma. Oncotarget, 9(29), p.20377. 
11.van den Boom, J., Wolter, M., Blaschke, B., Knobbe, C.B. and Reifenberger, G., 2006. Identification of novel genes associated with astrocytoma progression using suppression subtractive hybridization and real-time reverse transcription-polymerase chain reaction. International journal of cancer, 119(10), pp.2330-2338.

12.Awakura, Y., Nakamura, E., Ito, N., Kamoto, T. and Ogawa, O., 2008. Methylation-associated silencing of TU3A in human cancers. International journal of oncology, 33(4), pp.893-899.

13.Pastuszak-Lewandoska, D., Czarnecka, K.H., Migdalska-Sęk, M., Nawrot, E., Domańska, D., Kiszałkiewicz, J., Kordiak, J., Antczak, A., Gorski, P. and Brzeziańska-Lasota, E., 2014. Decreased FAM107A expression in patients with non-small cell lung cancer. In Respiratory Carcinogenesis (pp. 39-48). Springer, Cham. 


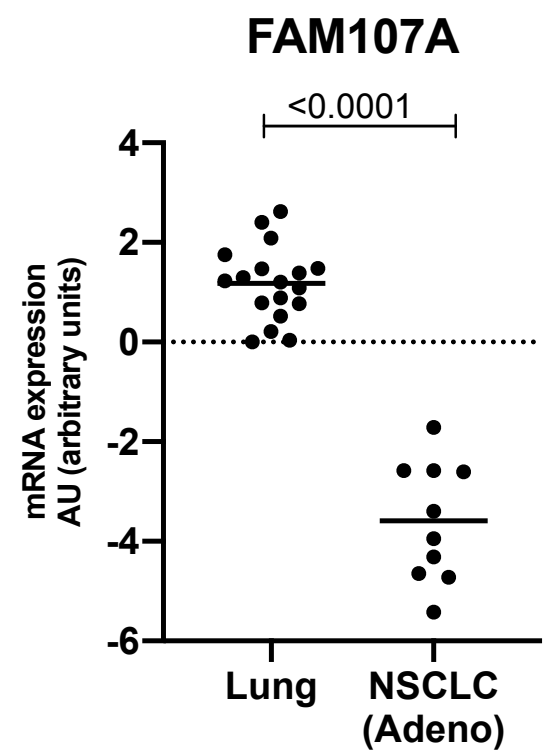

Figure 1: Fam107A is expressed at significantly lower levels in NSCLC tumors when compared to the lung.

The mRNA expression level of Fam107A is graphically represented in the lung (left) and in NSCLC tumors of the adenocarcinoma type (right) with mean mRNA expression values marked and the result of a statistical test evaluating significance of difference in Fam107A expression between NSCLC tumors and the lung, a $p$-value, listed above. 


\section{FAM107A}

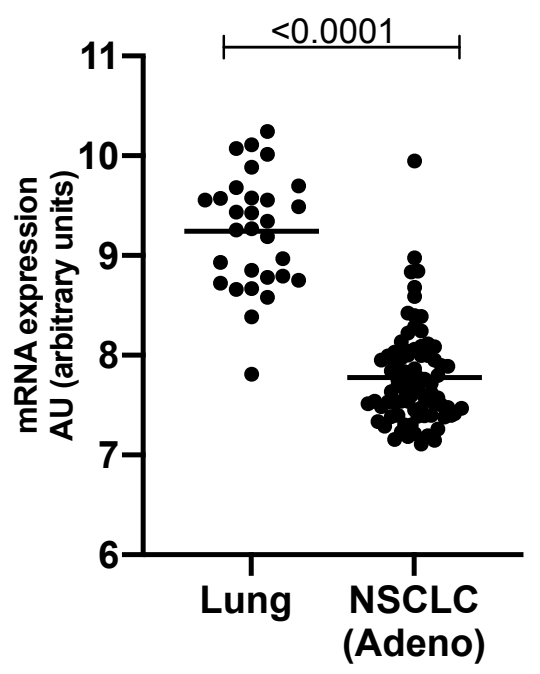

Figure 2: Fam107A transcript is expressed at significantly lower levels in NSCLC tumors when compared to the lung.

The mRNA expression level of Fam107A is graphically represented in the lung (left) and in NSCLC tumors of the adenocarcinoma type (right) with mean mRNA expression values marked and the result of a statistical test evaluating significance of difference in Fam107A expression between NSCLC tumors and the lung, a $p$-value, listed above. 


\section{FAM107A}

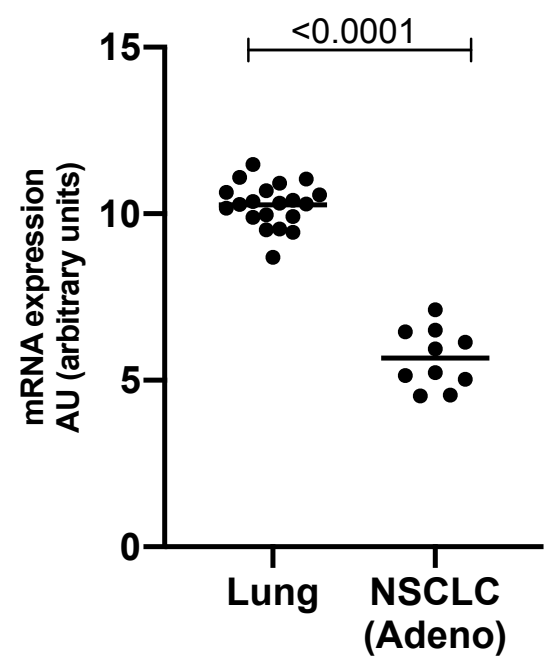

Figure 3: Fam107A transcript is expressed at significantly lower levels in NSCLC tumors when compared to the lung.

The mRNA expression level of Fam107A is graphically represented in the lung (left) and in NSCLC tumors of the adenocarcinoma type (right) with mean mRNA expression values marked and the result of a statistical test evaluating significance of difference in Fam107A expression between NSCLC tumors and the lung, a $p$-value, listed above. 
FAM107A (209074_s_at)

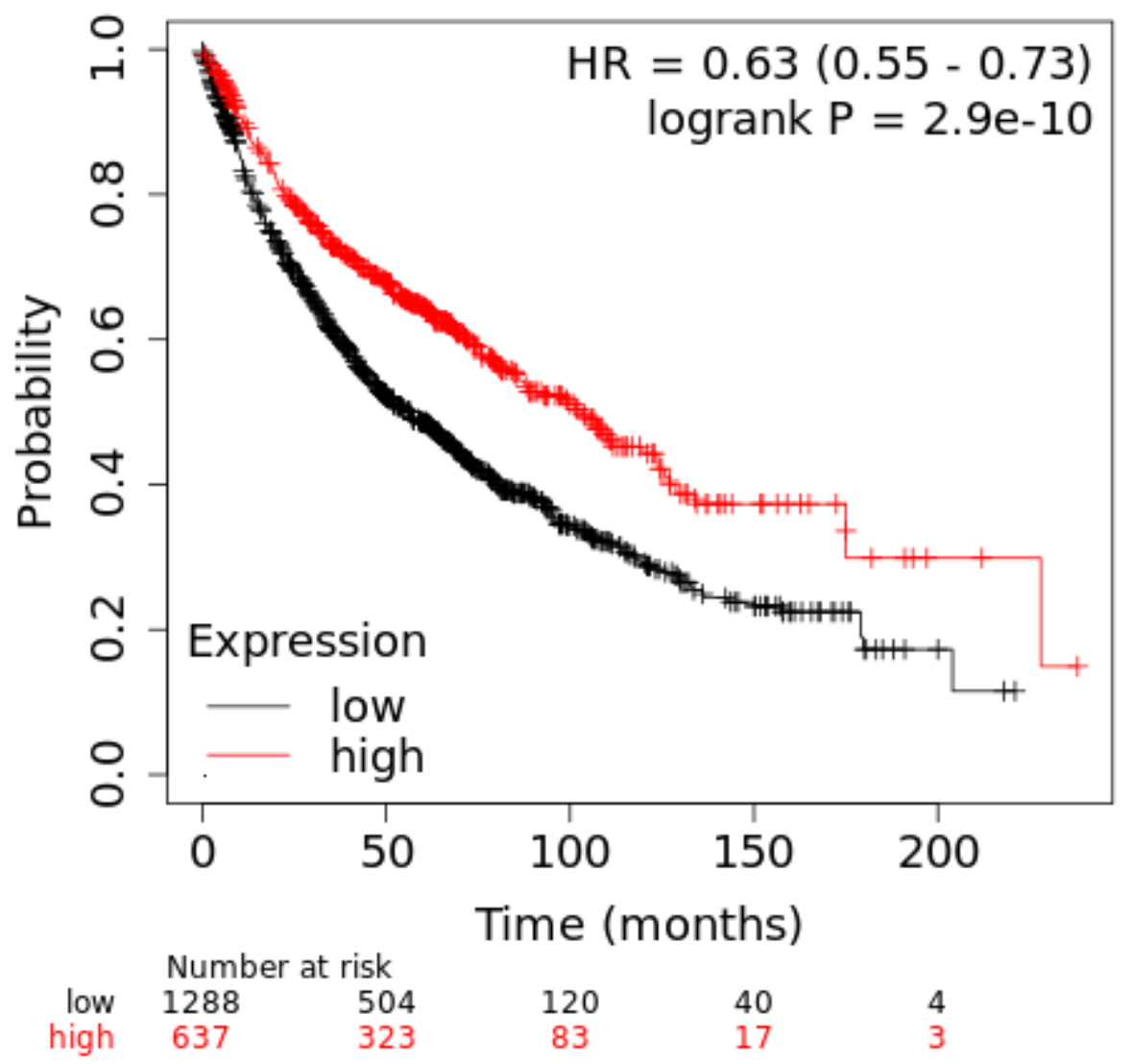

Figure 4: Fam107A expression in NSCLC tumors significantly correlates with overall survival.

Depicted in this Kaplan-Meier plot is the probability of overall survival for $n=1925$ total patients stratified into two groups, based on low or high expression of Fam107A in patient tumors. The $\log$ rank $p$-value denoting statistical significance of difference in overall survival when comparing the two groups, as well as hazard ratio for this comparison is listed above. Listed below is the number of patients at risk (number of patients alive) per interval, after stratification based on Fam107A expression; in the first interval, number at risk is number of patients alive; in each subsequent interval, number at risk is the number at risk less those who have expired or are censored. 

survival.

The median overall survival of $n=1925$ NSCLC patients based on stratification into low or high expression of Fam107A in tumors is listed in this chart. 103

Table 4: Fam107A expression in NSCLC tumors significantly correlates with patient 